\title{
Evaluation of Transducer and Signature Selections on the Performance of Artificial Intelligence Machine Tool Wear Prognosis
}

\section{I-Chun Sun}

Department of Mechanical Engineering, National Cheng-Kung University Tainan, Taiwan

\section{Renchi Cheng}

Department of Mechanical Engineering, National Cheng-Kung University Tainan, Taiwan

Kuo-Shen Chen ( $\nabla$ kschen@mail.ncku.edu.tw )

National Cheng-Kung University

\section{Research Article}

Keywords: Artificial Intelligence, Domain knowledge, Feature extraction, Sensor index evaluation, Tool condition assessment

Posted Date: August 10th, 2021

DOI: https://doi.org/10.21203/rs.3.rs-782301/v1

License: (c) (1) This work is licensed under a Creative Commons Attribution 4.0 International License. Read Full License 


\title{
Evaluation of Transducer and Signature Selections on the Performance of Artificial Intelligence Machine Tool Wear Prognosis
}

\author{
I-Chun Sun, Ren-Chi Cheng, and Kuo-Shen Chen** \\ Department of Mechanical Engineering, National Cheng-Kung University \\ Tainan, Taiwan, 70101, R.O.C. \\ ${ }^{* *}$ Corresponding Author: Email: kschen@mail.ncku.edu.tw
}

\begin{abstract}
The qualities of machined products are largely depended on the status of machines in various aspects. Thus, appropriate condition monitoring would be essential for both quality control and longevity assessment. Recently, with the advance in artificial intelligence and computational power, status monitoring and prognosis based on data driven approach becomes more practical. However, unlike machine vision and image processing, where data types are fixed and the performance index has already well defined, sensor selection and index for machine tools are versatile and not standardized at this moment. Without supporting of appropriate domain knowledge for selecting appropriate sensors and adequate performance index, pure data driven approach might suffer from unsatisfied prediction accuracy and needing of excessive training data, as well as the possibility of misjudgment. This would be a key obstacle for promoting data driven based prognosis in general intelligent manufacturing field. In this work, the status monitoring and prediction of a cutter wear problem is investigated to address the above concerns and to demonstrate the possible solutions by hiring a 5-axis machine center equipped with milling cutters of different wear levels. Transducers including accelerometers, microphones, current transformer, and acoustic emission sensors are mounted on the spindle, fixture, and nearby structures to monitor the milling process. The collected data are processed to extract various signatures and the key dominated indexes are identified. Finally, three multilayer perception (MLP) artificial neural network models are established. These models trained by different input features are compared to examine the influence of selected sensors and indexes on the prediction accuracy. The results show that with appropriate sensors and signatures, even with less amount of experimental data, the model can indeed achieve a better prediction. Therefore, a proper selection of indexes guided by physical knowledge based experiment or theoretical investigation would be critical.
\end{abstract}

Keywords-Artificial Intelligence, Domain knowledge, Feature extraction, Sensor index evaluation, Tool condition assessment

\section{INTRODUCTION}

Machine tools such as computer numerical control (CNC) milling machines play key roles in modern manufacturing and they usually represent the highest cost in the production lines
[1]. The quality of machined products is largely depended on the status of machines in various aspects [2]. As a result, appropriate condition monitoring would be essential for quality control, safety, and life assessment of machine tools especially for the current Industry 4.0 era [3]. There are numerous statuses related to machine tool conditions in various sub-systems ranged from driving motors, high speed spindles [4], lead screw and fixtures, and various cutting tools, as well as the cooling systems [5,6]. How to monitor their status and provide appropriate assessments in both healthy condition and life prediction would be a non-trivial task.

With recent advancement in computer science, artificial intelligence (AI) becomes an alternative choice for establishing condition diagnostic model. AI provides a decision-making system by using multiple sensor features to predict the states of machines in a data driven manner. For example, Li[7] used neural network and time/frequency domain bearing vibration analysis to carry out motor rolling bearing fault diagnosis. Su and Chong [8] proposed an analytical redundancy method using neural network modeling of the induction motor in vibration spectra for machine fault detection and diagnosis. Ghosh [9] developed a neural network-based sensor fusion model for tool condition monitoring. Essential features were extracted from a number of machining signals, namely cutting forces, spindle vibration, spindle current, and sound pressure level, and then the features were fused to estimate the main status such as average flank wear of milling cutters. Binsaeid [10] extracted 135 different features from multiple sensor signals of force, vibration, acoustic emission and spindle power in both time and frequency domains. Then, multiple machine learning (ML) and machine ensemble (ME) methods were used to classify tool conditions. While many research works focus on the adjustment of model parameters or trying different algorithm to improve accuracy, certain fundamental issues were still not reported. That is, although the AI approach has already been proved to be feasible in machine tool monitoring, several concerns arises. First, for a successful monitor/ diagnosis/ prognosis task, transducers are required. However, what transducers are suitable and where they should be installed? For example, if one placed an accelerometer at vibration node, it would not 
sense the response of a particular shape. Second, which kind of performance index should be used for data reduction to yield the most efficient result? These two concerns are not trivial. First, sensors and data acquisitions/processing represents additional costs imposed on the machine tools. Ineffective sensors represent a waste of investment and reduction in product competition. For example, Kuma et al indicated that acoustic emission sensor was ineffective and could even reduce the prediction rate during their investigation on transmission box failure diagnosis [11]. Second, with improper index, undesirable results such as excessive training and low prediction rate and even wrong conclusion might occur [12]. Appropriate domain knowledge should be integrated into the entire monitoring system architecture for avoiding the above two concerns [13]. However, these issues are seldom addressed in previous related works. Technically, the key of these two concerns is on the specific domain knowledge such as cutting dynamics, rotor dynamics, and tribology. With the associate domain knowledge and mathematical models in hands, in conjunction with essential understandings on sensors and signal processing, one should be able to address the above two concerns in an analytical manner. Consequently, the efficient and accuracy of AI-based diagnosis schemes could be improved.

This problem is not trivial since improper sensors and indexes could significantly increase the machine cost and might suffer from unsatisfied prediction accuracy and excessive training data, as well as the possibility of misjudgment. It is recommended that selection of sensors and index should have background in related domain knowledge. However, to the best of our knowledge, virtually all previous data-driven based machine status works ignored this issue and concentrated on the development of status prediction based on their selected sensors/indexes.

In short, there remains a gap on discussing the effect of selected sensors/indexes on the efficiency and accuracy of datadriven prediction. To address the above concern, in this work, the problem on wear status prediction of milling cutters during machining would be investigated as the platform using machine learning approach. Specifically, multilayer perception (MLP) artificial neural network (ANN) model [14], which has been widely used ranged from biomedical diagnosis [15] to structural reliability assessment [16], would be used. Various sensors are installed, and common performance indexes are extracted from the collected data. Suitable or unsuitable index are then explained using domain knowledge perspective. These data and indexes are then examined for their relevance. Finally, the efficiency and accuracy of MLP ANN models based on different input data (screened or unscreened) are then created and to compare with each other. By this approach, it is desired to highlight the importance of choosing appropriate sensors and index, as well as forming a rational decision flow, to the performance of AI predictions. This should provide an example for future machine monitoring activity based on AI approach.

To demonstrate the importance of the problem and to elucidate the key thought of this work, a relatively simple but typical machining scenario should be chosen, and the investigation flow is shown in Fig. 1. A mini 5-axis CNC machine tool serves as the main platform and several milling cutters with certain wear levels are hired for machining. Various transducers and high-speed data acquisition systems are then installed for collecting data. Through a designated experimental plan, the collected data are then used to evaluate the effectiveness of each performance index and to find the most effective sensors/index group. Finally, ANN-based diagnostic models are created and trained/validated by those cutting data with various subsets to evaluate the effectiveness of used sensors and performance sets in accuracy of wear status prediction. This result and the study procedure should be useful for determining the most critical sensors and performance index to be investigated for cost effective consideration.

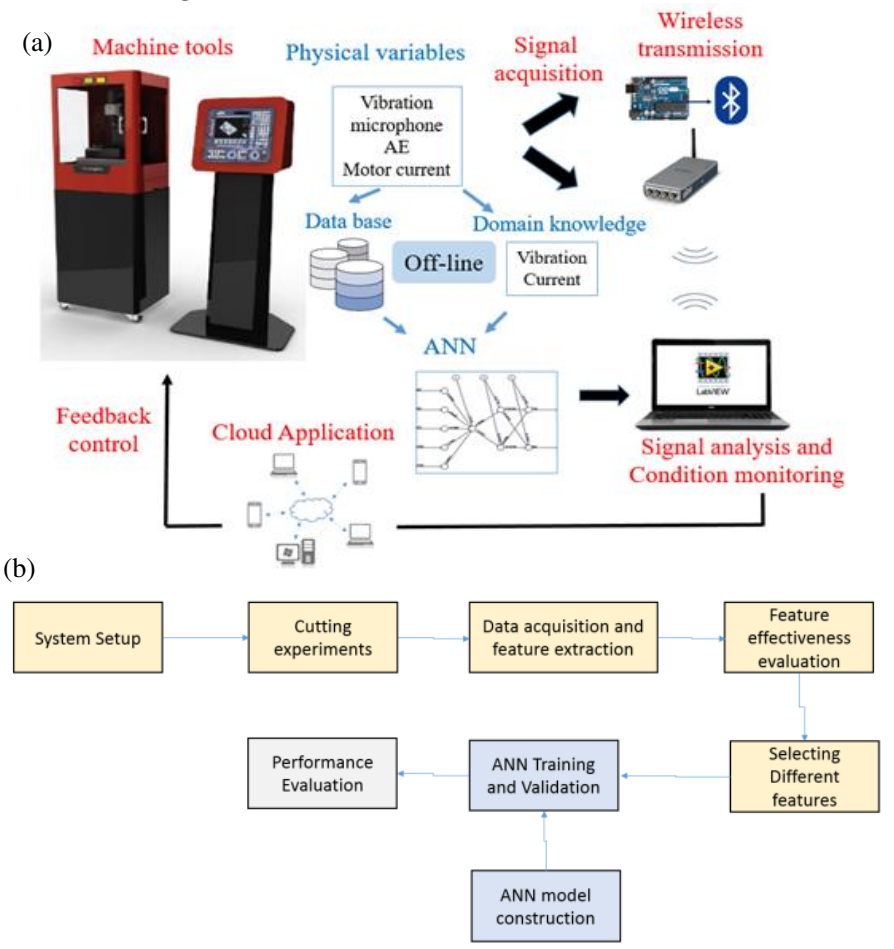

Fig. 1. The overall scope of this work (a) system layout and perspective, (b) the detail research flow.

Based on the first author's master thesis [17], the rest of this article presents the technical detail. In section II, the overall system setup and experiment plan is anchored and addressed for guiding the entire study. The characterization of the hired machine tool before machining is presented in Section III. Followed by the associated cutting experiments and the associated data acquisition, as well as the preliminary signatures extraction and their sensitivity evaluation addressed in Section IV. Consequently, In Section V, the signature set and the obtained test data are then used to develop various ANN-based wear prediction schemes for wear status diagnosis and for evaluating the needs of each transducer and the effectiveness of each performance index. The key discovery, contribution, and recommended future works are then discussed in Section VI. Finally, Section VII concludes this work. 


\section{SyStEM SETUP}

\section{A. Experimental system setup and general plan}

The first step is to design and realize the entire testing system. As shown in Fig. 2a, a miniaturized 5-axis PMC machine center is served as the core of the system. Several end milling cutters with different wear levels are then used. Transducers, including two piezoelectric accelerometers (PCB 353B16, Kistler 8763B050), one piezoelectric microphone (PCB 130F020), an acoustic emission transducer (Kistler 8152C003), and a current transformer, as well as the key information (such as rotating speed) provided by the corresponding machine controller are used to monitor the operation status. In addition, before conducting experiment, the wear conditions of these milling cutters are also inspected by an optical microscope. Relatively soft material blocks, such as aluminum T6061, brass, and hard epoxy, are then fixed on a vise for performing cutting. The rotating speed, depth of cutting, and feed rate, are the major controlling parameters. Detail specification of these transducers are list in Table 1. The detail sensor installation is shown in Fig. 2b. For acoustic emission, the sampling rate for data collection is $2 \mathrm{MHz}$. On the other hand, a $40 \mathrm{KHz}$ sampling rate is used for collecting data from other transducers. For each experiments, data are collected for 20 seconds.

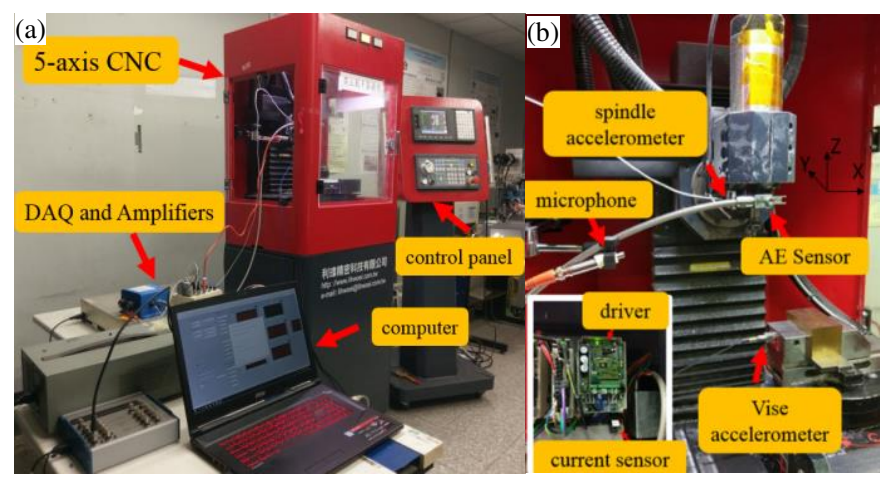

(c)

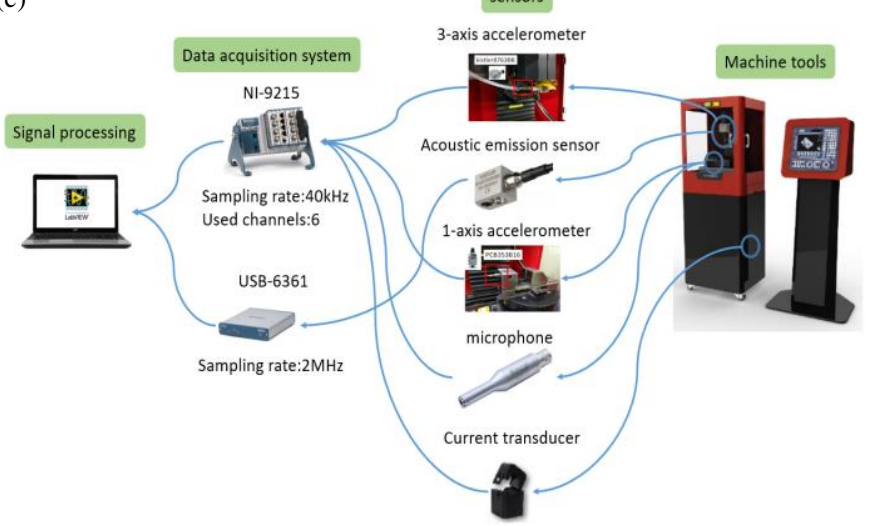

Fig. 2. The system setup for performing experiemnts: (a)(b) setup and (c) signal flow.

In this work, statistically measurements of sensor signal are taken for both time and frequency domains. A completed list of features used in vibration diagnosis could be found elsewhere $[4,11,18]$ and here only a few frequently used quantities in both time and frequency domains. Here time domain features including, mean $(\mu)$, standard deviation $(\sigma)$, root mean square $(r m s)$, kurtosis, and entropy. For a data set $x$, their definitions are listed in below.

$$
\begin{gathered}
\mu=\frac{1}{n} \sum_{i=1}^{n} x_{i} \\
\sigma=\sqrt{\frac{\sum_{i=1}^{n}\left(x_{i}-\mu\right)^{2}}{n-1}} \\
r m s=\sqrt{\frac{1}{n} \sum_{i=1}^{n} x_{i}{ }^{2}} \\
\text { Kurtosis }=\frac{1}{n} \frac{\sum_{i=1}^{n}\left(x_{i}-\mu\right)^{4}}{\sigma^{4}} \\
\text { entropy }=-\sum P\left(x_{i}\right) \log _{2} P\left(x_{i}\right)
\end{gathered}
$$

Where $\mathrm{P}\left(\mathrm{x}_{\mathrm{i}}\right)$ is the appearance probability density function. On the other hand, frequency domain features, such as resonance frequencies, crest ratio, ratio of power (ROP), and even octave band, can be used as the potential measures. The total power $P_{\text {total }}$ in a spectrum S(f) is expressed as [19]

$$
P_{\text {total }}=\int_{0}^{f_{S} / 2} S(f) d f
$$

Where $f_{s}$ is the sampling frequency. Finally, another frequently used indicator, ratio of power (ROP), is then defined as

$$
\mathrm{ROP}=\frac{\int_{f_{1}}^{f_{2}} S(f) d f}{P_{\text {total }}},
$$

which represents the power ratio in a particular interested frequency band (between $f_{1}$ and $f_{2}$ ) and is widely used in acoustic emission signal analysis.

Both time and frequency domain features have significant implications in machine tools monitoring. For exampl e, RMS represents the overall vibration level. In addition, Kurtosis is four central moments and commonly used in machine condition monitoring [20], because failures of machine components often excite relatively large pulses in the time domain waveform. On the other hand, frequency characteristics could be linked to certain operating parameters such as spindle rotating speed, chattering frequency, and even bearing damages.

In this investigation, there are totally 720 cutting experiments performed and the raw data are collected by these transducers and features, both in time and frequency domains, would be extracted. Based on parametric studies, the sensitivity of each signature is evaluated and discussed physically. Key signatures are then identified. Based on literature reviews and initial experimental investigations, a total of 29 features are selected as the key features for future artificial intelligence training. The corresponding control parameters for experiments are the initial wearing state of milling cutters, spindle rotating speed, cutting depth, and the materials for cutting. The entire information is listed in Table 2. Notice that these 29 features include effective and non-effective signatures and would be judged by the 
subsequent experimental and analysis investigations.

MLP ANN models are then developed. The models are feed and trained with different types of data and their corresponding performance index. Among them, $70 \%$ of the above-mentioned data are used to train and the rest $30 \%$ are used for validation. In particular, it is desired to evaluate the relationship between the selected signatures and the achieved prediction accuracy since this result would provide key information on determining appropriate transducers and data reduction schemes for optimizing subsequent machine learning schemes. That is, in this work, three ANN models would be created by feeding different training data set. The first one would be fed by data of all 29 signatures. On the other hand, the second one would be fed by six most effective performance index. Finally, the third model would be fed by indexes from one dominated sensor. The effectiveness of different machine learning model based on different chosen signatures would be investigated for elucidating the importance of selecting proper signal signatures based on both prediction accuracy and cost of investment.

TABLE 1. TRANSDUCERS USED IN THIS INVESTIGATION

\begin{tabular}{|c|c|c|}
\hline Transducers & Spec & Remarks \\
\hline $\begin{array}{l}\text { Kister 8763B050 3-axis } \\
\text { accelerometer }\end{array}$ & $\begin{array}{lrr}\text { bandwidth } & 10 \mathrm{kHz}, \\
\text { sensitivity } & 95-110 \\
\mathrm{mV} / \mathrm{g} & \end{array}$ & $\begin{array}{l}\text { w/ PCB } 584 \text { IEPE } \\
\text { charge amplifier }\end{array}$ \\
\hline $\begin{array}{l}\text { PCB 353B16 1-axis } \\
\text { accelerometer }\end{array}$ & $\begin{array}{l}\text { bandwidth } 10 \mathrm{kHz} \text {, } \\
\text { sensitivity } 10.3 \mathrm{mV} / \mathrm{g}\end{array}$ & $\begin{array}{l}\text { w/ PCB } 584 \text { IEPE } \\
\text { charge amplifier }\end{array}$ \\
\hline PCB 130F20 microphone & $\begin{array}{l}\text { bandwidth } 16 \mathrm{kHz} \text {, } \\
\text { sensitivity } 41.7 \mathrm{mv} / \mathrm{Pa}\end{array}$ & $\begin{array}{l}\text { w/ PCB } 584 \text { IEPE } \\
\text { charge amplifier }\end{array}$ \\
\hline $\begin{array}{l}\text { CTT-10-CLS-CV-5 } \\
\text { Current transformer }\end{array}$ & sensitivity $1 \mathrm{~V} / \mathrm{A}$ & \\
\hline $\begin{array}{l}\text { Kistler } 8152 \mathrm{C} 0 \text { Acoustic } \\
\text { emission sensor }\end{array}$ & $\begin{array}{l}\text { Suitable band } 50 \mathrm{k}- \\
400 \mathrm{kHz}, \quad \text { sensitivity } \\
57 \mathrm{dBref} 1 \mathrm{~V} /(\mathrm{m} / \mathrm{s})\end{array}$ & $\begin{array}{l}\text { w/ Kistler } 5125 \mathrm{C} 1 \\
\text { charge amplifier }\end{array}$ \\
\hline DAQ & $\begin{array}{l}\text { NI } 921540 \mathrm{kHz} / \mathrm{ch} \\
\text { NI USB } 6361,2 \mathrm{MHz}\end{array}$ & $\begin{array}{l}\text { USB } 6361 \text { is for } \mathrm{AE} \\
\text { sensor }\end{array}$ \\
\hline
\end{tabular}

TABLE 2. THE CONTROL AND KEY FEATURE INDEX FOR THE EXPERIMENT

\begin{tabular}{|c|c|}
\hline Sensors/ Controls & Features \\
\hline $\begin{array}{l}\text { Cutting Control } \\
\text { Parameters (total 3) }\end{array}$ & $\begin{array}{l}\text { Spindle rotating speed } \\
\text { Cutting depth } \\
\text { Initial wearing state from optical microscopy }\end{array}$ \\
\hline $\begin{array}{l}\text { 1-axis accelerometer } \\
\text { at vise platform (total } \\
\text { 4) }\end{array}$ & $\begin{array}{l}\text { RMS (1) } \\
\text { Kurtosis (1) } \\
\text { Spectrum at rotating frequency (1) } \\
\text { Spectrum at double rotating frequency (1) }\end{array}$ \\
\hline $\begin{array}{l}\text { 3-axis accelerometer } \\
\text { at spindle (total } 8)\end{array}$ & $\begin{array}{l}\text { RMS (3) } \\
\text { Kurtosis (3) } \\
\text { Spectrum at rotating frequency in } \mathrm{x} \text {-axis (1) } \\
\text { Spectrum at double rotating frequency in } \mathrm{x} \text {-axis (1) }\end{array}$ \\
\hline Microphone (total 4) & $\begin{array}{l}\text { RMS } \\
\text { Kurtosis } \\
\text { Spectrum at rotating frequency (1) } \\
\text { Spectrum at double rotating frequency (1) }\end{array}$ \\
\hline $\begin{array}{l}\text { Acoustic emission } \\
\text { sensor (total 9) }\end{array}$ & $\begin{array}{l}\text { RMS (1) } \\
\text { Kurtosis (1) } \\
\text { ROPs at } 7 \text { frequency bands }(\mathrm{kHz})(50-100,100 \text { - } \\
150,150-200,200-250,250-300,300-350,350-400)\end{array}$ \\
\hline $\begin{array}{l}\text { Current transformer } \\
\text { (total 2) }\end{array}$ & $\begin{array}{l}\text { Mean value (1) } \\
\text { Standard deviation }(1)\end{array}$ \\
\hline
\end{tabular}

\section{Design FOR CharacterizING CUtTING WeAR}

The wear status of a cutting tool is an important indicator in any metallic cutting process for estimating additional costs in terms of scrapped components, machine tool breakage and unscheduled downtime results [21]. Thus, how to proper monitor and predict their states of wear level is important. Previously, various analytical models $[22,23,24]$ and measurement techniques $[21,25]$ have been proposed beyond the original Taylor's equation [26] and this fact makes it being an ideal case as the benchmark problem for developing machine learning in tool status diagnosis.

For achieving the goal on wear prediction of milling cutters, a thoughtful experiment plan designed. Schematically shown in Fig. 3, slot milling is chosen in this work for maximizing the material usage efficiency. The feeding direction is set as $x$ direction. Relative soft materials T-6061 aluminum alloy is used as the cut materials since it will not cause significant wear on cutters. This could ensure the parameter control during experiments. Spindle rotating speed and the depth of cutting are selected as two major control variables.
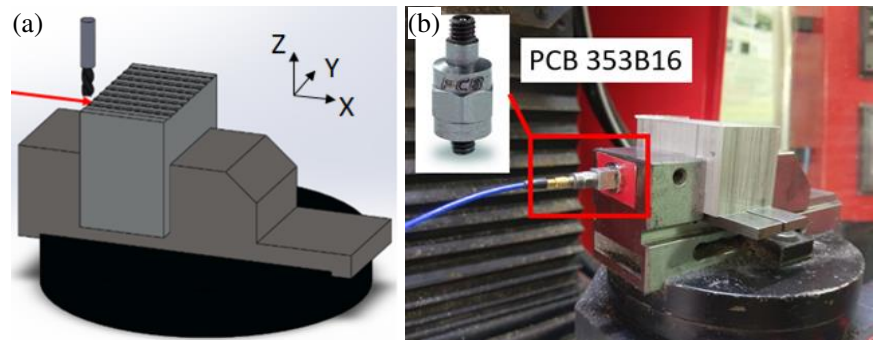

Fig. 3. The cutting experiments: (a) schematic plot and (b) setup.

The first step of the experiment is to create different wear levels in milling cutters. The experiments are conducted by using the CNC machine with tungsten carbide four-flute end milling cutters coated with TiAlN. To achieve controllable wear in short duration, brand new cutters are used to cut S45C medium carbon steel with a rotating speed $16000 \mathrm{rpm}$, cutting depth $0.3 \mathrm{~mm}$, and a feeding rate of $1300 \mathrm{~mm} / \mathrm{min}$. without cutting fluid or lubricants. In summary, a cutter would experience approach $40 \mathrm{k}$ cuttings for completely a single milling mission. Under such a harsh condition with a relative hard material, it is expected that the cutters can be wear out soon. Here we define the term "cutting index" as the number of milling missions and "slightly wear" when a cutter with a cutting index of 5, which equivalent to undergo approximately 200k cutting cycles with the above cutting condition and the term "seriously wear" for cutting index of 10 or the cutting cycle is approximately $400 \mathrm{k}$ cycles. Meanwhile, the real wear condition at cutter franks are also observed and measured by a CCD (BEST ETA, BV-1080) mounted optical microscopy (OPTEM, ZOOM 125C and M-Plan APO 10x) shown in Fig.4a. Typical frank wear pictures are also presented in Fig. $4 \mathrm{~b}$ for the purpose of comparison. Under microscope, the wearing zone can be easily identified. The characteristic width (i.e., $x$ ) and length (i.e., $y$ ) of wear zone can be measured from the acquired images. As shown in Fig.5, the wear levels can be completely separated in terms of wearing area $(x$ times $y)$. 

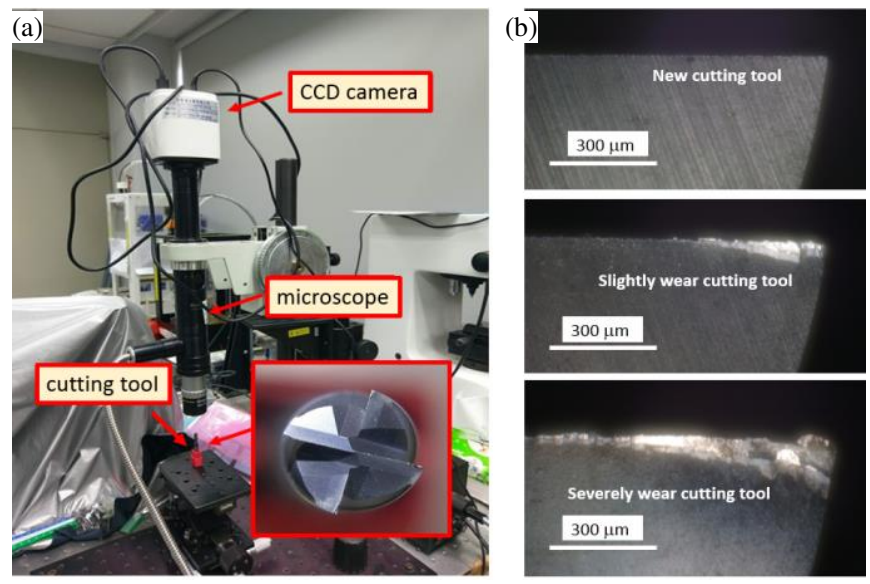

Fig. 4. (a)The vision-based tool wear sensing system setup and (b) the pictures of cutting tools at different wear conditions.

(a)

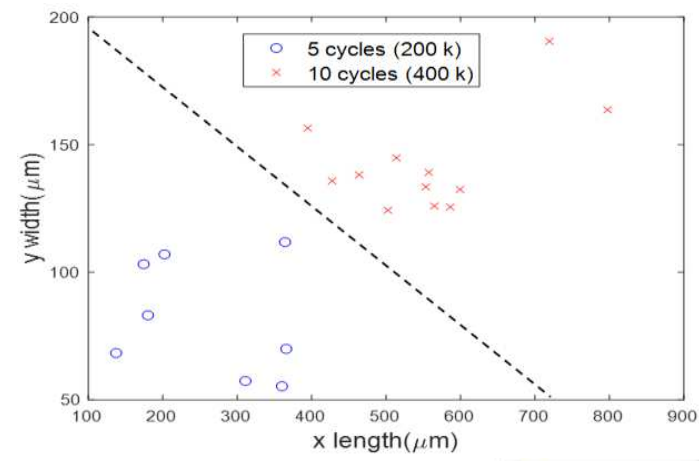

(b)

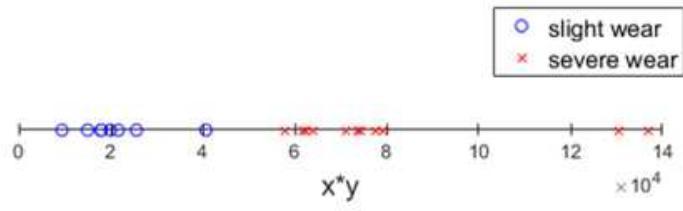

Fig. 5. (a)The relationship between cutter wear frank length $(x)$ and width $(y)$ at different cutting runs. Each cycle represents $40 \mathrm{k}$ cutting on medium carbon steel S45C and (b) data classification after manipulation by length and width multiplication.

Once the wear conditions are defined, milling experiment would then be proceeded. The experiments are conducted by the PMC 5-axis CNC miller for machining T-6061 aluminum alloy work piece using cutters with different wear levels. The experimental design is briefly summarized in Table 3 . After the data base is established, one can proceed signal analysis as mentioned previously to obtain feature indexes of each sensor.

TABLE 3. DESIGN OF CUTTING EXPERIMENT WITH CUTTING TOOL AT DIFFERENT WEAR CONDITION

\begin{tabular}{|c|c|c|c|c|}
\hline Spindle rotating speed & $8 \mathrm{k} \mathrm{rpm}$ & $9 \mathrm{k} \mathrm{rpm}$ & $10 \mathrm{k} \mathrm{rpm}$ & $12 \mathrm{k} \mathrm{rpm}$ \\
\hline Cutting depth & $0.2 \mathrm{~mm}$ & $0.1 \mathrm{~mm}$ & $0.1 \mathrm{~mm}$ & $0.2 \mathrm{~mm}$ \\
\hline Brand new trials & 60 & 60 & 60 & 60 \\
\hline Slight wear trials & 60 & 60 & 60 & 60 \\
\hline Serious wear trials & 60 & 60 & 60 & 60 \\
\hline
\end{tabular}

Fig. 6 shows typical vibration obtained at the platform (Fig. 6a) and at the spindle (Fig. 6b) accelerometers. It can be seen that both vibration levels increase with wear index. However, although the spindle has larger vibration level, the vibration measured at vise platform has a better sensitivity to the tool worn index. Meanwhile, the response of the microphone shown in Fig. 6c also exhibits similar behavior as that seen on the spindle accelerometer. Finally, one can see (in Fig. 7) both acoustic emission level and the motor current also vary w.r.t. wear level in certain sense.

After completing the machining experiments, the cutting signal data base is then established and one can proceed data reduction to investigate the relationship between major sensor indexes and cutting parameters at different tool conditions. As addressed in Section II, there are 29 key indexes (listed in Table 2 ) to be extracted. A systematic investigation is performed to evaluate the sensitivity and effectiveness of these index w.r.t. tool wear index, it is found that many of those sensor indexes either have no obvious correlations with tool wear index or have no sufficient sensitivity. Finally, after evaluation, six features (listed in Table 4) exhibiting either better correlation or stronger sensitivities are selected as a subset of optimal sensor indexes. Their behavior and correlations with tool wear would be detailed documented in Section IV and for subsequent ANN machine status prediction.
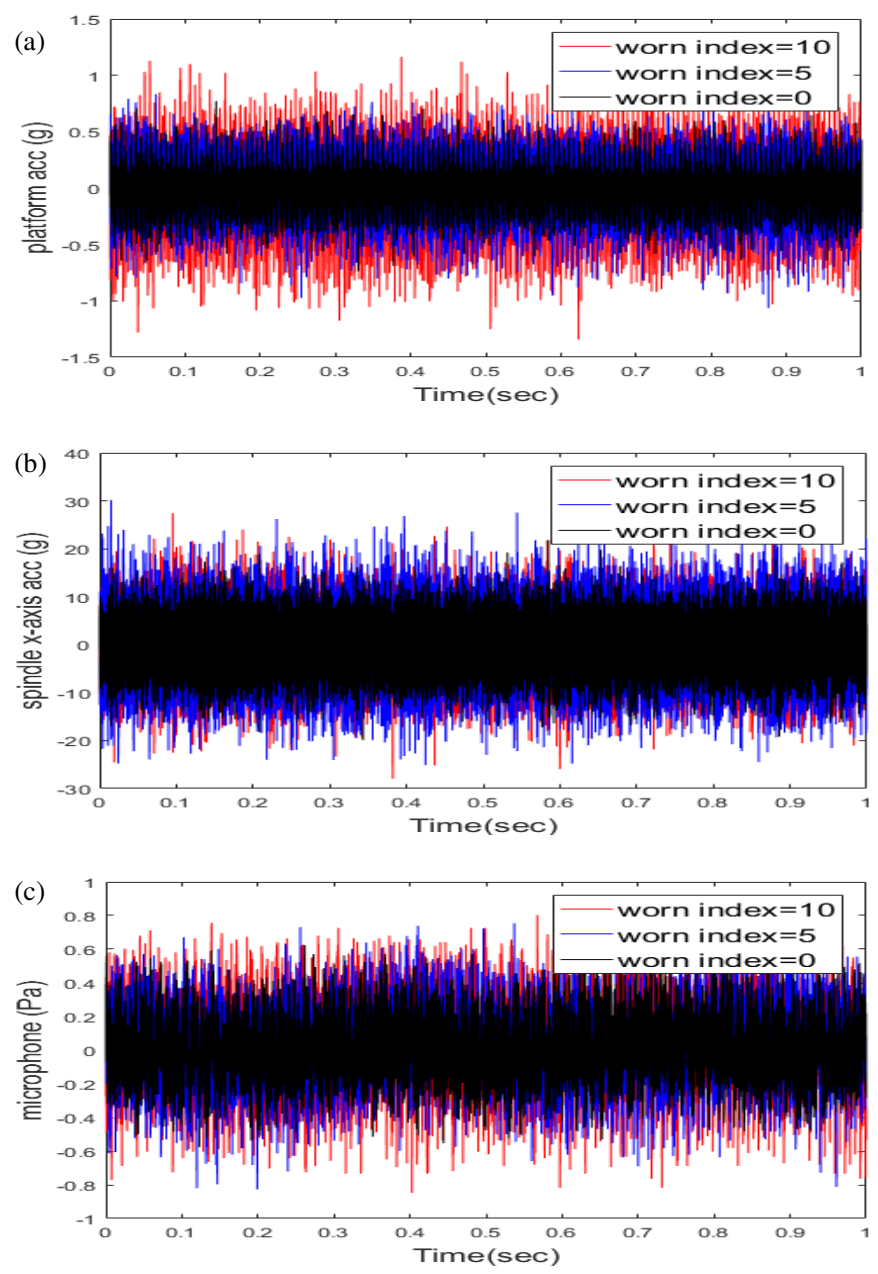

Fig. 6. Typical sensor responses during milling for different worn levels: (a) vise accelerometer, (b) spindle accelerometer, (c) microphone. 

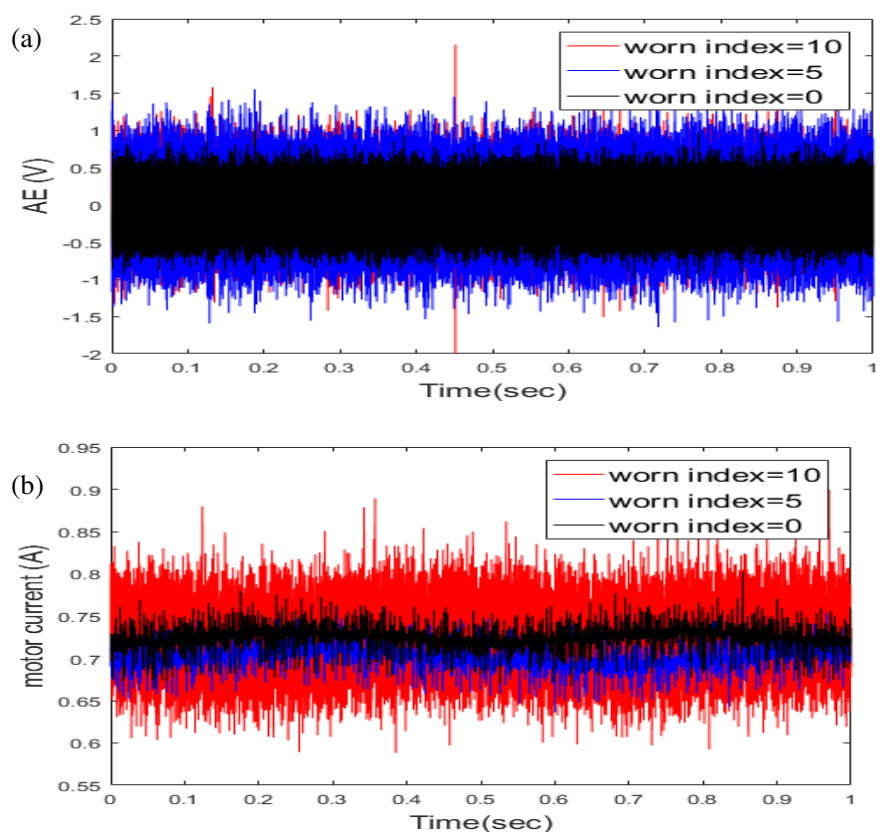

Fig. 7. Typical sensor responses during milling for different worn levels: (a) $\mathrm{AE}$ sensor and (b) current transformer

TABLE 4. THE MOST DOMINATED FEATURES FOR TOOL WEAR STUDY

\begin{tabular}{|l|l|}
\hline Sensors & Features \\
\hline $\begin{array}{l}\text { accelerometer at } \\
\text { platform (vise) }\end{array}$ & $\begin{array}{l}\text { RMS } \\
\text { Frequency spectrum at second harmonics }\end{array}$ \\
\hline Microphone & RMS \\
\hline $\begin{array}{l}\text { Acoustic emission } \\
\text { sensor }\end{array}$ & ROPs at (50-100) kHz and (100-150) $\mathrm{kHz}$ \\
\hline Current transformer & Mean value \\
\hline
\end{tabular}

\section{DATA REDUCTIONS FOR MACHINING EXPERIMENTS}

In this section, the data reduction results for the features listed in Table 4 are first presented. Fig. 8 shows the $r m s$ values of accelerations measured from the vise surface and of the microphone under different wearing conditions. It can be seen that both quantities and the tool wear index are highly correlated at different machining conditions. On the other hand, the acceleration measured at spindle, although the vibration level is much larger, it fails to show informative trends and is therefore not treated as an effective index. Meanwhile, the relationship between the spectrum strength of the second harmonics and the readout of the current transformer w.r.t. tool wear index are shown in Fig. 9. It can be seen that, in general, it has clear trends with different wear index and cutting parameters, however, the data is not so neat for particular machining parameters (i.e., in this case, cutting speed at $8000 \mathrm{rpm}$ and cutting depth $0.2 \mathrm{~mm}$ ). These indexes can be treated as partially effective. Finally, the ROPs of the AE sensors at different machining conditions at different bands are shown in Fig. 10. It can be seen that in most bands, there are no distinguishable difference except that at the bands of $50 \mathrm{kHz}-100 \mathrm{kHz}$ and 100 $\mathrm{kHz}-150 \mathrm{kHz}$. In fact, there are many indexes shows poor correlations such as kurtosis and entropy, which are frequently used as the performance index in previous works [4, 27, 28]. On the other hand, motor current and sound, as suggested in previous work [29], exhibit meaningful correlation. These six most influential indexes are listed and is shown in Table 4 and they will be used as the major training input for ANN tool conditional monitoring to evaluate the effect of sensor/ feature selection on the accuracy of ANN prediction. Notice that all major indexes are only partial effective, without subsequent AI modeling, it is challenging to develop accurate predictions based on the correlations between sensor indexes and tool wear conditions.
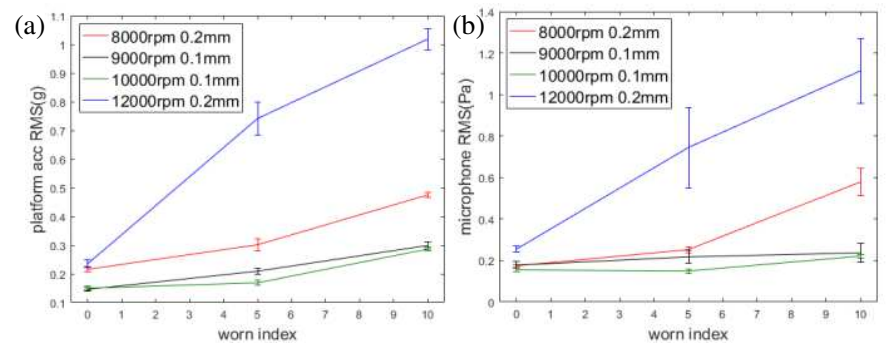

Fig. 8. RMS results of (a) platform (vise) vibration and (b) microphone output. (a)
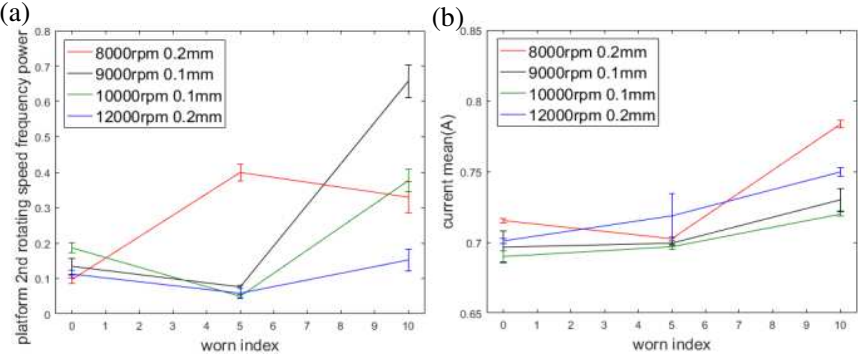

Fig. 9. (a) second rotating speed frequency power of platform(vise) vibration and (b)mean value of current transducer output.
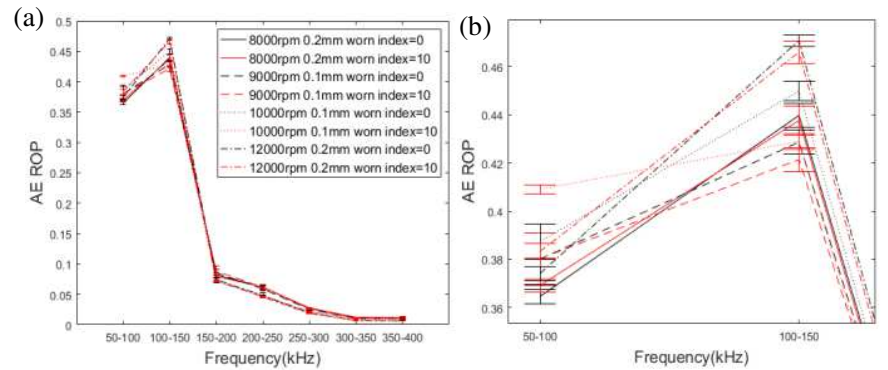

Fig. 10. ROP of AE sensor (a)all frequency bands (b)local frequency bands

\section{DeVEloping ANN-BASED Status Diagnosis}

After finishing the experimental work and define performance indexes, the next issue is to construct the intelligent status monitoring system for identifying the abnormalities using signatures of collected data based on a decision-making system. For this purpose, the well-recognized artificial neural network technique will be hired [9]. In the future, other possible methods based on both supervised and unsupervised learning techniques will be followed [30,31]. Fig. 11 shows the schematic flow on the entire training and validation procedure of performing ANN 
investigations. Here, has already mentioned, in addition to use typical reported sensing indexes, the developed ANN model would also be trained by the most effective indexes screened in Section IV. It is hope that by pervious physical-based performance index screening, the accuracy of prediction can be reduced since it is impractical and difficult to accumulate huge number of cutting data for ANN training. There are a few tasks should be accomplished to establish an ANN-based diagnostic model, including model design, model training and model testing. The results are going to be performed below.

\section{A. ANN model design}

The utilized ANN model is essentially a MLP with back propagation algorithm. Fig. 12 shows a typical MLP ANN model architecture with back propagation. Basically, an ANN model contains an input layer with $\boldsymbol{n}$ inputs, an output layer with $\boldsymbol{m}$ outputs and $\boldsymbol{q}$ hidden layers, which contains $\boldsymbol{p}_{\boldsymbol{i}}$ neurons ( $i=1$ to $q$ ) for each hidden layers. In addition, several adequate activation functions have been used inside the neuron. As a result, $\boldsymbol{n}, \boldsymbol{m}, \boldsymbol{q}, \boldsymbol{p}_{\boldsymbol{i}}$, and the selected activation functions must be pre-determined for constructing ANN models. In this work, depending on the model requirement, $\boldsymbol{n}$ is chosen as 8,29 , or 3 . On the other hand, $\boldsymbol{m}$, and $\boldsymbol{q}$ are fixed as 3 and 2. Meanwhile, ReLu, Sigmoid, and Softmax functions are used to link layers. Cross-entropy is chosen as the measure of the loss function. During each training, the output results are compared with standard training data and the residual error is used to adjust the weighting coefficient in the ANN model through proper optimizer such as Adam. Such a process continues until the accuracy satisfied. The entire model structure is shown in Fig. 13. The models are created using Spyder with Tensorflow [32] and Keras [33] as the application program interface under Anaconda environment. All models are executed under Window 10 64bit system with an i-9 CPU and 64G memory, and a NVIDIA GeForce GTX 1050 GPU with 35G memory.

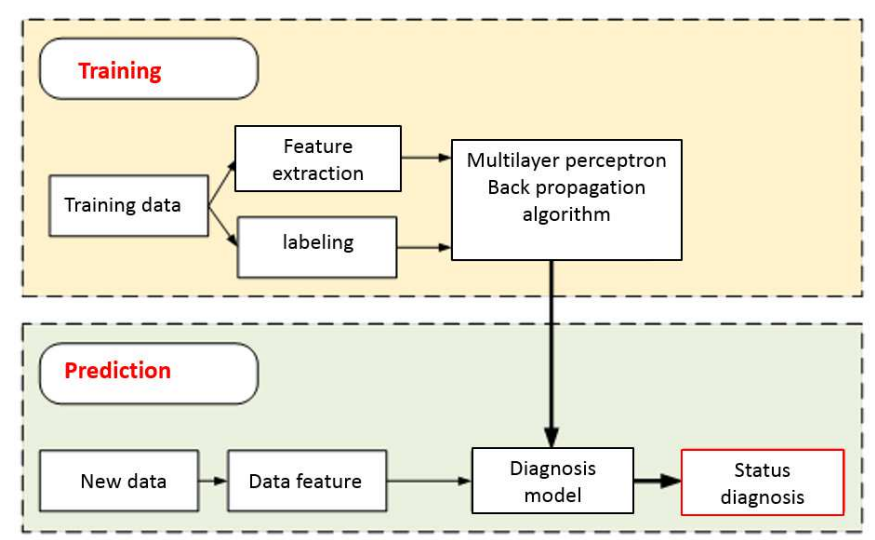

Fig. 11. Schematic flow for training and prediction of MLP ANN model.

Once the model is generated, it would experience a series of parametric studies for determining the required numbers of neurons. Such a process continues until the accurate rate satisfied. Taking the case with 8 inputs as the example to explain the training condition, the result is shown in Fig. 14. It can be seen that the training accuracy rises as the neurons number increases and remains constant after the neurons number is over 25 (Fig. 14(a)). However, as shown in Fig. 14(b), the number of epochs needed to successfully train the model is that the number of hidden layer neurons is over 70. As a result, the neurons numbers (i.e., $\boldsymbol{p}_{1}$ and $\boldsymbol{p}_{2}$ ) at two hidden layers are set to 100 in this study. The final MLP model structure in this work is already shown in Fig. 12.

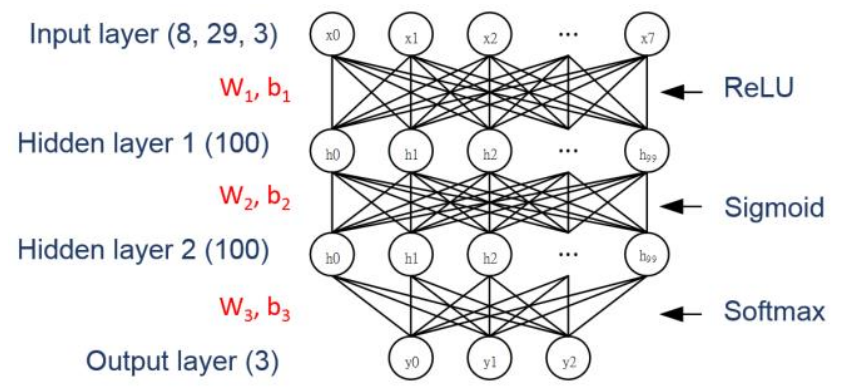

Fig. 12. Schematic plot of the final MLP model structure.

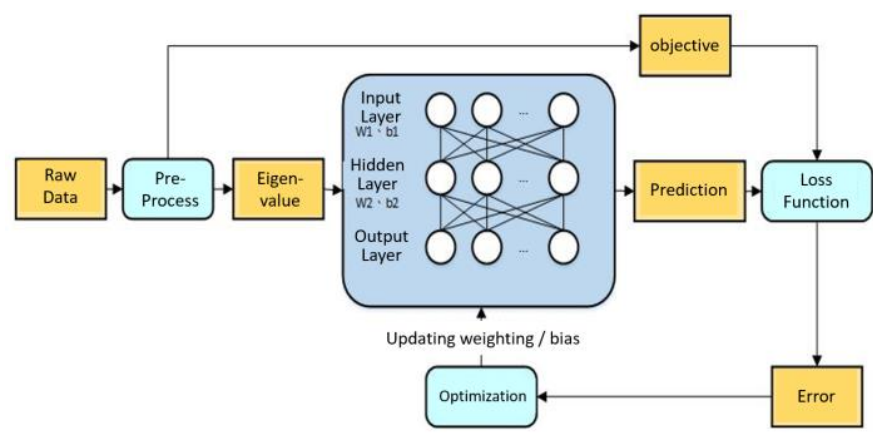

Fig. 13. The overall ANN modeling flow

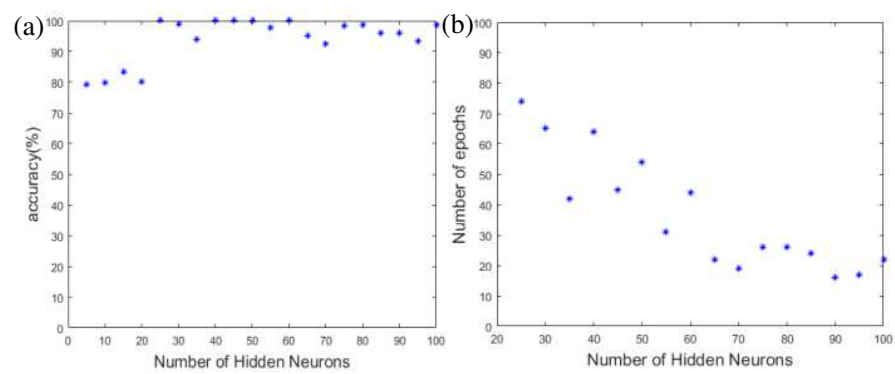

Fig. 14. Examination of the number of hidden layer neurons (a)with training accuracy (b)with number of epochs.

\section{B. Training and assessment results}

After constructing the MLP ANN models, training and validations are followed. As shown in Sections III and IV, there are total 720 sets of data from cutting experiment with different operating and wear conditions. As addressed before, among them, 70 percent data are used for training models and 30 percent are utilized to validate their accuracy. In order to examine the effect of chosen indexes, three different combinations of sensor feature indexes are set to be model inputs for training. One (model I) is aimed for demonstrating the effect of appropriate performance index (i.e., the six most sensitive indexes), another (model II) is to evaluate the effect of hiring more unscreened indexes. Finally, the last one (model III) is to evaluate the successful rate in prediction by using 
indexes from just a most effective sensor. Practically, this issue is not trivial since installing more sensors implies the increase of capital cost. If the successful rate of prediction is fair with only one sensor and one index, this could be the most favorable approach from practical concern.

Detail information of these three sets is listed in Table 5. Model 1 consists of 8 effective feature indexes based on the evaluation in Section IV, including six indexes from sensor index evaluation and two cutting parameters: rotating speed and depth of cut. On the other hand, model 2 is composed of all feature indexes from five sensors and two cutting parameters as well and there are totally 29 indexes. In spite of high accuracy for tool condition assessment, it needs more sensor indexes to train the model, that could make this model not attractive for the purpose of cost reduction. Finally, model 3 is trained by two cutting parameters and only one sensor index, i.e. $r m s$ value of the vise platform acceleration.

The training evolution for the three model is shown in Fig. 15 . It can be seen that for model 1 , it requires approximately 30 epochs to reach the accuracy level, which is near $100 \%$. However, slight overfitting is also observed. The overall prediction accuracy is approximately $98.7 \%$ and only 3 wrong prediction out of 228 pieces of testing data. For model 2, as shown in Fig. 15, it only requires 20 training epochs to achieve near $100 \%$ accuracy. However, the aftermath overfitting is more severe than that of model 1 . In validation phase, there are 20 wrong predictions out of 206 testing data set and an accuracy of $90.3 \%$ is achieved. Finally, for model 3, as shown in Fig. 15, it can be found that training accuracy can only be converged to $90 \%$. For validation, there are 45 wrong predictions out of 214 test data sets and an accuracy of only $78.9 \%$. The confusion matrices of these three models are all listed in Table 6.

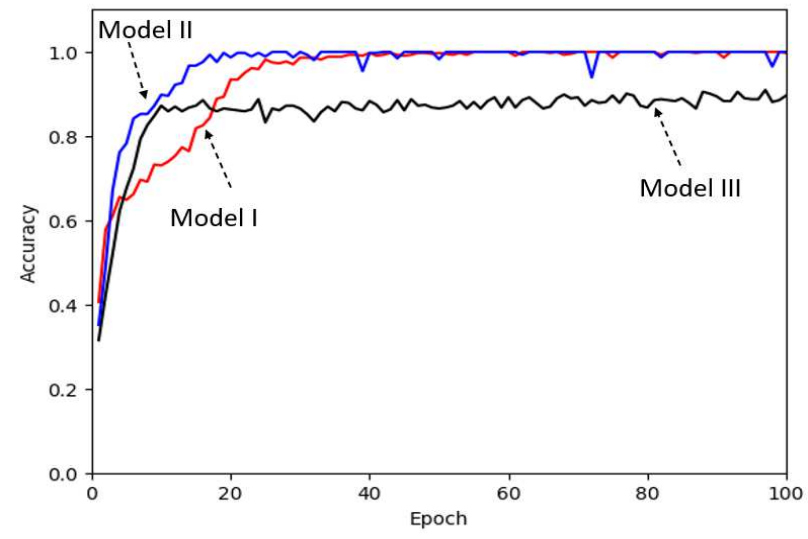

Fig. 15. The relationship between training accuracy vs. training epoch for all three models.

TABLE 5. THE INPUT FEATURES DESCRIPTION AND TESTING RESULT FOR EACH MODEL

\begin{tabular}{|l|l|c|}
\hline & \multicolumn{1}{|c|}{ Number of input features } & accuracy \\
\hline Model 1 & $\begin{array}{l}\text { 8 (6 sensor indexes listed in Table 4), } \\
\text { cutting depth, rotating speed }\end{array}$ & $98.7 \%$ \\
\hline Model 2 & $\begin{array}{l}\text { 29 (all 27 sensor indexes list in Table 2), } \\
\text { and cutting depth, rotating speed }\end{array}$ & $90.3 \%$ \\
\hline Model 3 & $\begin{array}{l}\text { 3(rms of the platform acceleration), cutting } \\
\text { depth, rotating speed }\end{array}$ & $78.9 \%$ \\
\hline
\end{tabular}

TABLE 6. THE CONFUSION MATRICES OF EACH MODEL.

\begin{tabular}{|c|c|c|c|c|c|c|c|c|c|c|c|}
\hline \multicolumn{4}{|c|}{ Model 1} & \multicolumn{4}{|c|}{ Model 2} & \multicolumn{4}{|c|}{ Model 3} \\
\hline Predict & 0 & 1 & 2 & Label & 0 & 1 & 2 & Predict & 0 & 1 & 2 \\
\hline 0 & 75 & 3 & 0 & 0 & 54 & 20 & 0 & 0 & 50 & 14 & 0 \\
\hline 1 & 0 & 78 & 0 & 1 & 0 & 67 & 0 & 1 & 9 & 65 & 0 \\
\hline 2 & 0 & 0 & 72 & 2 & 0 & 0 & 65 & 2 & 0 & 22 & 54 \\
\hline
\end{tabular}

The above results indicate the importance of selecting proper sensors and performance index on achieved predicting successful rate. Although model 3 needs less input features, the accuracy rate of $78.9 \%$ is poor and may not be applicable for real applications. Based on the evaluation result, model 1 has the best prediction accuracy but it requires four different sensors. It also successfully confirms the feasibility to integrate domain knowledge and AI for improving the efficiency of model construction. Hence, this concept can be further applied to other sub-systems which are also lack of physical models for establishing status diagnostic model in the future.

On the other hand, model 2 has a less inferior accuracy but it requires all five sensors and 27 indexes, which represents a significant capital and computational cost. This implies that without proper screening to identify key controlling indexes, by means of significant amount of data only might not achieve satisfied results. Essential domain knowledge should be helpful to achieve better results with less experimental and computational cost. Finally, model 3 has the poorest accuracy but it only requires one sensor. Thus, this made this model cost effective. From practical industrial perspective, if the $80 \%$ accuracy is acceptable (for some low-level tasks), this model might be preferred.

The above investigation clearly indicates the importance on selecting suitable sensors and suitable performance index on the final prediction accuracy of machine learning models. Thus, we believe that it is important to address the reasons on choosing particular sensors and particular performance indexes before preceding specific machine learning model construction and training. However, this issue is actually seldom addressed in previous reported articles.

\section{DISCUSSIONS}

\section{A. A short summary}

In this work, machine learning based on artificial neural network method is applied to predict the wear level of milling cutters. A mini- CNC five-axis machine is used to conduct the cutting experiments on brass. There are totally five sensors (two accelerometers, an acoustic emission sensor, a microphone, and a current transformer) are hired and in total 27 performance indexes are used to predict the status of the cutting tools based on 720 cutting experiments. The sensitivity of each index is systematically evaluated as a basis for identifying key 
controlling index. ANN models based on three different sets of indexes are performed to examine the effect of selected performance indexes on the accuracy of status prediction. It is found that with proper selection of key indexes, the prediction accuracy can be better than using more indexes. This conclusion agrees with the preliminary observation by Kuma et al [11]. As a result, how to properly select sensor indexes would be crucial for machine learning efficiency and cost. Meanwhile, the study also shows that just use a major index may not be able to achieve a sufficient accurate prediction ( $80 \%$ ). However, depends on application, if such an accuracy level is acceptable, such a single sensor/single index model would have the strongest potential from industrial application perspective.

To the best of our knowledge, most works on machine leaning for machine status prediction usually focus on machine leaning method and its process but did not investigate the above-mentioned issue and usually did not provide firm reasons for sensor and index selections. As one has seen above, choosing appropriate index could be crucial for subsequent ANN prediction. Therefore, we believe that the major contribution of this work is on filling such a gap to provide fundamental information on the importance of properly selecting sensor index and its associate development flow to achieve a successful machine learning prediction.

\section{B. Key discovery and discussions}

Here a short comment on physical base of selected sensor indexes is addressed. First, in this work, it is observed that the measured acceleration of the spindle accelerometer is not so important and effective than that obtained from the platform (i.e., on the vise) accelerometer. As a result, the spindle accelerations are all excluded from the dominated sensor indexes. This is as expected since the cutting vibration is directly transferred from the work piece to the vise. On the other hand, the spindle vibration generated on the bearings are shielded, attenuated, and complicated by the casing, in which the accelerometer is mounted. It is believed that if one can directly install accelerometers at the vicinities of bearings, the situation could be very different. As a consequence, design and realize tiny acceleration measurement device inserting inside spindle is important and is actually our current work [34].

Second, spindle motor current measurement is always an effective method. From electromechanical coupling, electric current can be an indirect measurement of torque and the different wear level during cutting would require different motor torque for machining. From engineering perspective, this measurement is also cost effective and straightforward. The observation agrees with the recommendation [29].

Furthermore, microphone can catch essential structural vibration (usually in middle or high frequency regimes) information radiated or leaked from sources such as contact bearing and cutting pieces. As a result, it is also a widely used sensor [29]. In particular, its bandwidth is usually higher than that of accelerometers. Certain high frequency noise generated could be detected. However, it would detect virtually all acoustic noise from both sources and surroundings. As a result, under a noisy and harsh environment, the original sound data should be further processed to extract the information of focused frequency band.

Finally, acoustic emission represents extremely high frequency elastic wave propagation which is resulted from fracture or deformation of hard or stiff materials and structures. Traditionally, it has been widely to use to measure the cutting of brittle materials and contact failure of hard ball bearings [35]. The current experiment results indicate that $\mathrm{AE}$ signal strength could be useful for certain frequency bands but not so effective in general due to cutting soft ductile materials. With improper signature, the correlation between $\mathrm{AE}$ signal and the wearing state may not be proper revealed. A knowledge on the formation mechanism of acoustic emission shall be helpful for identifying key index for proper monitoring in different applications.

\section{Focused future works}

This work hires ANN to predict the wearing status of milling cutters and to illustrate the importance of selecting proper sensors and indexes for the subsequent machine learning predictions. In order to further improve the presented, the following works are currently investigated or scheduled to be the important future work.

As addressed above, the spindle accelerometer is less effective than the one mounted on the vise. It is believed that if it can be further miniaturized to fit the size constraint and is mounted at the vicinity of bearing, more critical information can be obtained, and the subsequent status prediction could be more reliable. Instead of using traditional piezoelectric accelerometers, a proper integration based on MEMS accelerometers should be able to satisfy both size and performance requirements. This serves as our immediately goal [34]. Furthermore, in addition to the used MLP ANN scheme, other supervised or unsupervised machine learning schemes such as autoencoder [36] would also be investigated in the future to further strengthen the argument of this work. Finally, it is desired to integrate process dynamics and mechanics into machine learning scheme [37] for enhancing the accuracy of prediction over the pure data driven approach. With other current investigated facility such as wireless communication and edge computation based smart factory data decision system ready [38,39], it is possible to feedback the analyze information to control the operation of machines for enhancing system reliabilities and for improving stability in milling and tunning processes [40,41].

\section{SUMMARY AND CONCLUSION}

Machine tools play key roles in modern manufacturing. The status monitoring and reliability, as well as the longevity prediction becomes essential for product quality insurance and cost saving. This work presents our initial effort toward developing infrastructures for conducting the above mentioned 
goals. Key issues such as low cost wireless data acquisition, signal processing, domain knowledge investigation, and development of ANN based assessment model, which is combined with the results of sensor index evaluation, are performed and addressed in this work. In addition, six most sensitive sensor indexes are listed for better describing the status of cutting tool. The system is realized via Arduino, Bluetooth, LabVIEW and Python. Finally, the developed system and the proposed concept are applied to tool condition assessment on a five-axis CNC miller for examining the feasibility. Meanwhile, in comparison with the other two model, the model trained by the results of sensor index evaluation obtain better performance.

In the near future, the performance of the presented system would be further increased for exploring new applications. Furthermore, with more data collected, it is expected that more sophisticated models based on domain knowledge would be developed for better describing and predicting the tool condition. Finally, the concept and the research flow in this work not only can be applied to tool condition assessment, but also can be employed by other subsystems for generating condition assessment models in the future to enhance the manufacturing reliability.

\section{ACKNOWLEDGMENT}

This work is supported by the Ministry of Science and Technology (MOST) of Taiwan under the contract numbers: 109-2221-E-006-096, and 109-2622-8006-005.

\section{REFERENCES}

[1] S. Kalpakjian and S. R. Schmid, Manufacturing Process for Engineering Materials, Prentice Hall, 2008.

[2] P. Stavropoulos, D. Chantzis, C. Doukas, A. Papacharalampop and G. Chryssolouris, "Monitoring and Control of Manufacturing Processes: $a$ Review," Procedia CIRP, vol. 8, pp. 421-425, 2013.

[3] M. Rüßmann, M. Lorenz, P. Gerbert, M. Waldner, J. Justus, P. Engel, and M. Harnisch, "Industry 4.0: The Future of Productivity and Growth in Manufacturing Industries" Boston Consulting Group, 2015.

[4] N. K. Hsieh, W. Y. Lin and H. T. Young, "High-speed spindle fault diagnosis with the empirical mode decomposition and multiscale entropy method," Entropy, vol. 17, pp. 2170-2183, 2015.

[5] P. Prickett and C. Johns, "An Overview of Approaches to End Milling Tool Monitoring", International Journal of Machine Tools and Manufacture, vol. 39, pp.105-122, 1999.

[6] R. Teti, K. Jemielniak, G. O'Donnell and D. Dornfeld, "Advanced Monitoring of Machining Operations," CIRP Annals-Manufacturing Technology, vol. 59, pp. 717-739, 2010.

[7] B. Li, M.-Y. Chow, Y. Tipsuwan, and J.C. Hung, "Neural-network-based motor rolling bearing fault diagnosis," IEEE transactions on industrial electronics, vol. 47, no. 5, pp. 1060-1069, 2000.

[8] H. Su and K.T. Chong, "Induction machine condition monitoring using neural network modeling," IEEE Transactions on Industrial Electronics, vol. 54, no. 1, pp. 241-249, 2007.

[9] N. Ghosh, Y. Ravi, A. Patra, S. Mukhopadhyay, S. Paul, A. Mohanty, and A. Chattopadhyay, "Estimation of tool wear during CNC milling using neural network-based sensor fusion," Mechanical Systems Signal Processing, vol. 21, no. 1, pp. 466-479, 2007.

[10] S. Binsaeid, S. Asfour, S. Cho, and A. Onar, "Machine ensemble approach for simultaneous detection of transient and gradual abnormalities in end milling using multisensor fusion," Journal of Materials Processing Technology, vol. 209, no. 10, pp. 4728-4738, 2009.
[11] T. P. Kumar, M. Saimurugan, R. B. Hari Haran, S. Siddharth and K. I. Ramachandran," A multi-sensor information fusion for fault diagnosis of a gearbox utilizing discrete wavelet features," Measurement Science and Technology, vol. 30, 085101, 2019.

[12] N. Lee, M. Azarian, and M. Pecht, "Octave-band Filtering for Convolutional Neural Network-based Diagnostics for Rotating Machinery," Proc. Annual Conference of the PHM Society, vol. 12, no. 1, pp. 9-9, 2020.

[13] N. Lee, M. Azarian, and M. Pecht, "Octave-band Filtering for Convolutional Neural Network-based Diagnostics for Rotating Machinery," in Proc. Annual Conference of the PHM Society, vol. 12, no. 1, pp. 9-9), 2020

[14] M. R. Meireles, P. E. Almeida, and M. G. Simões, "A comprehensive review for industrial applicability of artificial neural networks," IEEE Transactions on Industrial Electronics, vol. 50, pp. 585-601, 2003.

[15] M. Salah, K. Altalla, A. Salah, and S. S. Abu-Naser, "Predicting Medical Expenses Using Artificial Neural Networ,". International Journal of Engineering and Information Systems (IJEAIS), vol. 2, pp. 11-17, 2018.

[16] W. C. Wu et al, "Development of Artificial Neural Network and Topology Reconstruction Schemes for Fan-Out Wafer Warpage Analysis," Proc. IEEE 71 ${ }^{\text {st }}$ Electronic Components and Technology Conference, July 2021.

[17] I-C Sun, Development of artificial neural network based status monitoring systems for tool condition assessment of CNC millers, Master Thesis, National Cheng-Kung University, Taiwan, 2019.

[18] B. Sick, "On-line and indirect tool wear monitoring in turning with artificial neural networks: a review of more than a decade of research," Mechanical systems signal processing, vol. 16, no. 4, pp. 487-546, 2002.

[19] C.H. Martins, P.R. de Aguiar, A. Frech, and E.C. Bianchi, "Tool Condition Monitoring of Single-Point Dresser Using Acoustic Emission and Neural Networks Models," IEEE Transactions on Instrumentation and Measurement, vol. 63, pp. 667-679, 2014.

[20] Y. Wang, J. Xiang, R. Markert, and M. Liang, "Spectral kurtosis for fault detection, diagnosis and prognostics of rotating machines: A review with applications," Mechanical Systems and Signal Processing, vol. 66, pp. 679-698, 2016.

[21] D. E. D. Snr, "Sensor signals for tool-wear monitoring in metal cutting operations - a review of methods," International Journal of Machine Tools and Manufacture, vol. 40, pp. 1073-1098, 2000.

[22] Y. Koren, "Flank wear model of cutting tools using control theory," Journal of Engineering for Industry, vol. 100, no. 1, pp. 103-109, 1978.

[23] E. Usui, T. Shirakashi, and T. Kitagawa, "Analytical prediction of cutting tool wear," Wear, vol. 100, pp. 129-151, 1984.

[24] H. Shao, H. Wang, and X. Zhao, "A cutting power model for tool wear monitoring in milling," International Journal of Machine Tools Manufacture, vol. 44, no. 14, pp. 1503-1509, 2004.

[25] J. Jurkovic, M. Korosec, and J. Kopac, "New approach in tool wear measuring technique using CCD vision system," International Journal of Machine Tools Manufacture, vol. 45, pp. 1023-1030, 2005.

[26] F. W. Taylor, On the Art of Cutting Metals, 3rd ed., The American Society of Mechanical Engineers, New York, USA, 1906.

[27] J. Antoni and R. B. Randall, "The spectral kurtosis: application to the vibratory surveillance and diagnostics of rotating machines," Mechanical Systems and Signal Processing, vol. 20, pp. 308-331, 2006.

[28] R. Yan and R. X. Gao, "Approximate entropy as a diagnostic tool for machine health monitoring," Mechanical Systems and Signal Processing, vol. 21, pp. 824-839, 2007.

[29] D. Salgado and F. Alonso, "An approach based on current and sound signals for in-process tool wear monitoring," International Journal of Machine Tools Manufacture, vol. 47, pp. 2140-2152, 2007.

[30] S. B. Kotsiantis, I. Zaharakis, and P. Pintelas, "Supervised machine learning: A review of classification techniques," Emerging artificial intelligence applications in computer engineering, vol. 160, pp. 3-24, 2007.

[31] M. Usama et al., "Unsupervised machine learning for networking: Techniques, applications and research challenges," IEEE Access, 2019, 7: 65579-65615.

[32] B. Ramsundar and R. B. Zadeh, TensorFlow for Deep Learning: from Linear Regression to Reinforcement Learning, O'Reilly Media, 2018.

[33] A. Gulli and S. Pal, Deep Learning with Keras, Packt Publishing Ltd, 2017. 
[34] J. M. Tsai, I. C. Sun, and K. S. Chen, "Realization and Performance Evaluation of a Machine Tool Vibration Monitoring Module by Multiple MEMS Accelerometer Integrations," International J. Advanced Manufacturing Technology, Vol. 114, pp.465-479, 2021.

[35] S. P. Gross, J. Fineberg, M. Marder, W. D. McCormick and H. L. Swinney, "Acoustic emissions from rapidly moving cracks," Physical Review Letters, 71(19), 3162, 1993.

[36] K. Han, Y. Wang, C. Zhang, C. Li, and C. Xu, "Autoencoder inspired unsupervised feature selection," In 2018 IEEE International Conference on Acoustics, Speech and Signal Processing (ICASSP), pp. 2941-2945, 2018.

[37] B. Luo, H. Wang, H. Liu, B. Li, and F. Peng, "Early fault detection of machine tools based on deep learning and dynamic identification," IEEE Transactions on Industrial Electronics, vol. 66, pp. 509-518, 2019.

[38] I. C. Sun and K. S. Chen, K. S. "Development of signal transmission and reduction modules for status monitoring and prediction of machine tools," Proc. 56 $6^{\text {th }}$ Annual Conference of the Society of Instrument and Control Engineers of Japan (SICE), pp. 711-716, Sep. 2017.

[39] W. L. Chen, C. T. Huang, K. S. Chen, L. K. Chang, and M. C. Tsai, "Design and Realization of Microcontroller-Based Remote Status Monitoring System for Smart Factory Task Planning Applications," 60th Annual Conference of the Society of Instrument and Control Engineers of Japan (SICE) (Submitted).

[40] D. Olvera, A. Elías-Zúñiga, O. Martínez-Romero, L. N. López de Lacalle, H. Martínez-Alfaro, H. R. Siller, and M. W. Pineda, "Improved predictions of the stability lobes for milling cutting operations of thin-wall components by considering ultra-miniature accelerometer mass effects," Int J Adv Manuf Technol vol. 86, pp. 2139-2146, 2016.

[41] G. Urbikain, D. Olvera, L. N. López de Lacalle, A. Beranoagirre, A. ElíasZuñiga, "Prediction methods and experimental techniques for chatter avoidance in turning systems: a review," Appl Sci vol.9, p. 4718, 2019.

\section{Declarations}

Ethics approval: Not applicable.

Consent to participate: Not applicable.

Consent for publication: All the authors agree with the publication.

Conflict of interest: The authors declare that they have no conflict of

interest.

Availability of data and material: Available upon request.

Code availability: Available upon request.

Funding: Please refer to the acknowledgement section. 